\title{
Evaluation of Antagonistic Potential of Bacillus subtilis against Plant Pathogenic Fungi
}

\author{
V. P. Kendre*, G. K. Giri and S. S. Mane \\ Department of Plant Pathology, Dr. Panjabrao Deshmukh Krishi Vidhyapeeth, \\ Akola (M.S.), India \\ *Corresponding author
}

\section{A B S T R A C T}

\section{Keywords}

Bacillus subtilis, Alternaria, Colletotrichum, Myrothecium, Fusarium, Rhizoctonia, Phoma, Curvularia, Sclerotium

Article Info

Accepted:

15 September 2020 Available Online: 10 October 2020
The purpose of this research was to evaluate antagonistic activity of Bacillus subtilis isolated from Phylloplane and rhizospheric soil of different field crops. Bacteria are able to synthesis a wide range of secondary metabolites with fungicidal capabilities. The antagonistic potential of Bacillus subtilis was assessed in vitro against some foliar and soil borne plant pathogenic fungi. The result indicated that tested phylloplane Bacillus subtilis isolates exhibited antagonistic potential in the range of 76.13$85.59 \%$ against foliar pathogenic fungi and also showed that rhizosphere Bacillus subtilis recorded between 57.16- $71.89 \%$ against soil borne pathogenic fungi. Thus results indicated that tested bacterial species can be used as promising biocontrol agent.

\section{Introduction}

Biological control is an environmentallyfriendly alternative to chemical pesticides and it is an attractive method protecting the plants from pathogens, because the wide usage of chemicals has a negative impact on the environment and human health. Many biocontrol agents were isolated by screening of the large number of soil or plant-associated microorganisms for antagonism against phytopathogens in vitro or in planta (Berg et al., 2001). A number of bacterial species with antagonistic activity were isolated from the rhizosphere of different plants. Among those, bacilli and pseudomonads are the most common isolates. It is known that various species of the Bacillus genus are able to stimulate the plant growth (Cazorla et al., 2007). Bacteria can promote plant growth through a number of mechanisms, such as improvement of plant nutrition; induction of systemic resistance; toxicity to pests and antagonism pathogens. The antagonistic 
activity of Bacillus is associated with the synthesis of various antimicrobial peptides, secreted enzymes, proteins and volatile organic compounds (VOCs). Many Bacillus isolates were shown to have antifungal activity against phytopathogenic fungi that make them good biocontrol candidates Cyclic lipopeptide fengycin plays an important role in this process (Mardanova et al., 2017). The strains of the species B. subtilis can vary considerably both phenotypically and genetically and that affects their antagonistic potential. Comparative analysis of proteomes of two B. subtilis strains with antagonistic potential highlighted the major differences in the composition of intracellular and extracellular proteins (Tan et al., 2013), some of which can be associated with antimicrobial properties. Because of their fast growth, ability to effectively grow in low cost media and to sporulate under undesirable conditions Bacillus isolates are the attractive candidates for application as the biocontrol agents. There is a growing demand for such agents since it is expected that global market for biopesticides will significantly expand in the next 3 - 5 years (Wu et al., 2015).

The aim of this study was to directly compare two antagonistic strains of Bacillus subtilis isolated from phylloplane and rhizosphere soil of different field crops, in their ability to suppress phytopathogenic fungi through production of cyclic lipopeptides, hydrolytic enzymes and siderophores.

\section{Materials and Methods}

\section{Collection and isolation of $B$. subtilis isolates}

Thirty isolates of $B$. subtilis were collected from different phylloplane and rhizosphere soil samples of cotton, soybean, pegionpea, paddy, green gram, sorghum, bean and chickpea, crops of Vidarbha region of
Maharashtra agro ecosystem by serial dilution technique on nutrient agar medium and designated as (PBs-1 to 15 and RBs-1-15) simultaneously.

\section{Collection of diseased samples for isolation of foliar and soil borne fungal pathogens}

Collected diseased crop plant samples from various field crops are as follows.

\section{In vitro screening of Bacillus subtilis isolates against plant pathogenic fungi}

The isolates of $B$. subtilis were evaluated in vitro for their antagonistic properties against foliar and soil borne fungal pathogen of different field crops, by using dual culture technique. The bio-agent and the pathogen were inoculated side by side in a single Petri plate containing solidified PDA medium. Three replications were maintained for each treatment with one control by maintaining only pathogen. The plates were incubated for 7 days at $28 \pm 1{ }^{\circ} \mathrm{C}$. The mycelial diameter of pathogen was measured in two directions and average was recorded. Per cent inhibition of growth of test pathogen was calculated using the following equation (Vincent, 1927).

Where;

$\mathrm{I}=\frac{\mathrm{C}-\mathrm{T}}{\mathrm{C}}$

$\mathrm{I}=$ Per cent inhibition of mycelium

$\mathrm{C}=$ Growth of fungal mycelium in control.

$\mathrm{T}=$ Growth of fungal mycelium in treatment.

\section{Results and Discussion}

\section{Collection of phylloplane and rhizospheric samples and isolation of Bacillus subtilis}

Plant and soil samples were collected from phylloplane and rhizosphere of kharif and 
rabi crops viz. cotton, soybean, pigeonpea, sorghum, green gram, paddy, and chickpea etc. from various locations of Vidarbha region of Maharashtra (Table 1). Collected samples were processed in the laboratory for isolation of Bacillus subtilis on nutrient agar medium by serial dilution technique.

Milky white colonies were observed on nutrient agar medium which after three days of incubation picked and streaked on fresh nutrient agar medium for pure culture and used for further study. The data presented in Table 2 and 3, Plate 1 shows the crop wise isolates of $B$. subtilis obtained from phylloplane and rhizospheric samples of different field crops.

The isolates are obtained confirme the existence of $B$. subtilis in the phylloplane of different crops which have been reported earlier by many workers. Patro et al., (2002) recorded three isolates of phylloplane bacteria (Plb) (Bacillus spp.) from mungbean leaves. Brian (2004) isolated and reported that Bacillus subtilis most abundant bacteria cultured from the phylloplane of soybean. Mohammadipour et al., (2009) studied the characterization of surfactin producing 290 phylloplane isolates of $B$. subtilis which was collected from different ecological zones of Iran. Similarly, Theodulos et al., (2003) reported that Bacillus subtilis as a natural inhabitants of the tomato phylloplane. Killani et al., (2011) isolated Bacillus subtilis from the soil sample collected from cowpea rhizosphere. Jadhav et al., (2014) studied five isolates of Bacillus subtilis from rhizospheric soil of wilt infected pigeonpea plants by serial dilution method.

Amin et al., (2015) collected fifty soil samples from different sites of Bahmanshir riverside in Abadan city, Iran and analyzed for presence of Bacillus species. Abdalla et al., (2015), Saha et al., (2015) and Ashwini and Srividya (2016) isolated Bacillus subtilis from the rhizosphere of tomato, egg plant and chilli. Mardanova et al., (2017) isolated Bacillus strains from the rhizosphere soil of potato roots and Smitha et al., (2017) also isolated Bacillus culture CaB5 and Bacillus subtilis strain $\mathrm{CcB} 7$ from the chickpea and pigeonpea rhizospheric soil by serial dilution plate technique. A total of five Bacillus spp. (TB1, TB2, TB3, TB4 and TB5) were isolated from rhizhospheric soil samples of tomato as reported by Karthick et al., (2017) and also thirty isolates of $B$. subtilis collected from different rhizosphere soil samples of chilli, chickpea, cotton, groundnut, onion, marigold, mustard, niger, pegionpea, paddy, sorghum, sunflower and wheat of Bagalkot, Ballari, Raichur and Koppal parts of North Eastern Karnataka by serial dilution and plate count technique on Nutrient agar medium (Rajkumar et al., 2018).

\section{Isolation of fungal pathogens}

Isolation of different fungal pathogens from diseased phylloplane and rhizospheric samples was done on PDA by tissue isolation method.

\section{Efficacy of Bacillus subtilis isolates against plant pathogenic fungi by dual culture technique}

\section{Efficacy of phylloplane Bacillus subtilis isolates against foliar fungal pathogens}

Data presented in the Table 5 and 6 indicated that all the isolates inhibited the growth of all tested foliar fungal pathogen in the range of 76.13 - 85.59\% against PBs13 isolate. The minimum mycelial growth $(12.50 \mathrm{~mm})$ with maximum mycelial growth inhibition (81.81\%) of Myrothecium roridum, Colletotrichum dematium, Alternaria alternata (soybean), Colletotrichum truncatum, Curvularia lunata, Phoma 
medicaginis and Alternaria alternata (cotton) was recorded in $B$. subtilis isolate PBs13 followed by PBs3 (72.81\%), PBs14 (69.45\%) and PBs4 (67.34\%) respectively. The other isolates of B. subtilis i.e. PBs15, PBs10, PBs6 and PBs7 were found least effective against all the fungal pathogens.

The results of present investigations are in agreement with the findings of Amaresan et al., (2012) who reported B. subtilis isolates were effective against Colletotrichum capsici in chilli. However, the suppression of mycelial growth of Colletotrichum gloeosporiodes causing anthracnose in Dendrobium with crude extract of antifungal compound produced by $B$. subtilis was also reported by Prapagdee et al., (2012). The inhibition of Colletotrichum lindemuthianum causing anthracnose of cowpea with different strains of B. subtilis (Bs-21, Bs-22 and Bs-23) was recorded by Adebanjo and Bankole (2004). Laha and Venkantaraman (2001), Muralidharan et al., (2004) and Singh and Sinha (2004) reported inhibition of Curvularia lunata causing black kernel in rice with B. subtilis $(97.77 \%)$.

Table.1 Particulars of collected diseased samples

\begin{tabular}{|c|c|c|c|c|}
\hline $\begin{array}{c}\text { Sr.No } \\
\text {. }\end{array}$ & Crops & $\begin{array}{l}\text { Diseased plant } \\
\text { part used for } \\
\text { isolation }\end{array}$ & Symptoms observed & $\begin{array}{l}\text { Fungal pathogens } \\
\text { Isolated }\end{array}$ \\
\hline 1 & \multirow[t]{2}{*}{ Cotton } & Leaves & $\begin{array}{l}\text { Lesion with concentric necrotic } \\
\text { rings with sporodochia }\end{array}$ & Myrothecium roridum \\
\hline 2 & & Leaves & $\begin{array}{l}\text { Small,circular } \\
\text { necrotic, spot }\end{array}$ to irregular, & Alternaria alternata \\
\hline 3 & Pigeon- pea & Root & $\begin{array}{l}\text { Purple band extending upward } \\
\text { from the base of stem, split root } \\
\text { observed }\end{array}$ & Fusarium udum \\
\hline 4 & \multirow[t]{3}{*}{ Soybean } & Leaf & Small, circular, necrotic, spot & Alternaria alternata \\
\hline 5 & & Leaf & $\begin{array}{l}\text { Brown, irregularly shaped spots } \\
\text { on stem, pods and petioles }\end{array}$ & Colletotrichum truncatum \\
\hline 6 & & Root & $\begin{array}{l}\text { Leaves and stem straw coloured, } \\
\text { root dark, shraded brittle and } \\
\text { devoid of lateral roots }\end{array}$ & Rhizoctonia bataticola \\
\hline 7 & \multirow[t]{2}{*}{ Sorghum } & Seed & Grain black colour & Curvularia lunata \\
\hline 8 & & Seed & $\begin{array}{l}\text { Fluffy white or pinkish } \\
\text { colouration }\end{array}$ & Fusarium moniliformae \\
\hline 9 & \multirow[t]{2}{*}{ Chickpea } & Root & $\begin{array}{l}\text { Drooping of petioles, rachis and } \\
\text { leaflet }\end{array}$ & Fusarium oxysporum f.sp. ciceri \\
\hline 10 & & Root & $\begin{array}{l}\text { Lower portion of stem is affected, } \\
\text { Decortication, mycelium and } \\
\text { mustard like sclerotia on stem }\end{array}$ & Sclerotium rolfsii \\
\hline 11 & Green gram & Leaf & $\begin{array}{l}\text { Small, enlarged dark brown to } \\
\text { blackish spot }\end{array}$ & Phoma medicaginis \\
\hline 12 & Paddy & Stem & $\begin{array}{l}\text { Oval or elliptical or irregular } \\
\text { greenish grey spot }\end{array}$ & Rhizoctonia solani \\
\hline 13 & Bean & Leaf & $\begin{array}{l}\text { Small watery, dark green to black } \\
\text { spots on pods and leaves }\end{array}$ & Colletotrichum dematium \\
\hline
\end{tabular}


Table.2 Isolates of phylloplane Bacillus subtilis obtained from different kharif and rabi crops

\begin{tabular}{|c|c|c|c|c|}
\hline $\begin{array}{l}\text { Sr. } \\
\text { No. }\end{array}$ & Crops & Districts & Locations & $\begin{array}{l}\text { B. subtilis isolates } \\
\text { designated as }\end{array}$ \\
\hline 1 & \multirow{2}{*}{ Cotton } & Wardha & Hinganghat & PBs1 \\
\hline 2 & & Yavatmal & Murali & PBs2 \\
\hline 3 & \multirow[t]{3}{*}{ Soybean } & Washim & Amkheda & PBs3 \\
\hline 4 & & Washim & Brahamanwada & PBs4 \\
\hline 5 & & Yavatmal & Yavatmal & PBs5 \\
\hline 6 & \multirow{2}{*}{ Pigeonpea } & Buldhana & Ganeshpur & PBs6 \\
\hline 7 & & Washim & Doangaon & PBs7 \\
\hline 8 & \multirow[t]{2}{*}{ Sorghum } & Akola & $\begin{array}{l}\text { Sorghum Research Unit, Dr. } \\
\text { P.D.K.V., Akola }\end{array}$ & PBs8 \\
\hline 9 & & Amravati & RRC, Amravati & PBs9 \\
\hline 10 & \multirow[t]{2}{*}{ Green gram } & Akola & Patur & PBs10 \\
\hline 11 & & Amravati & Warud & PBs11 \\
\hline 12 & \multirow[t]{2}{*}{ Paddy } & Bhandara & Sakoli & PBs12 \\
\hline 13 & & Gondia & Amgaon & PBs13 \\
\hline 14 & \multirow[t]{2}{*}{ Chickpea } & Akola & Akola & PBs14 \\
\hline 15 & & Wardha & Dhanora & PBs 15 \\
\hline
\end{tabular}

Table.3 Isolates of rhizospheric Bacillus subtilis obtained from different kharif and rabi crops

\begin{tabular}{|c|c|c|c|c|}
\hline $\begin{array}{l}\text { Sr. } \\
\text { No. }\end{array}$ & Crops & Districts & Locations & $\begin{array}{l}\text { B. subtilis isolates } \\
\text { designated as }\end{array}$ \\
\hline 1 & \multirow[t]{3}{*}{ Cotton } & Amaravati & Paratwada & RBs1 \\
\hline 2 & & Buldhana & Bhadgaon & RBs2 \\
\hline 3 & & Nagpur & $\begin{array}{c}\text { College of } \\
\text { Agriculture,Nagpur }\end{array}$ & RBs3 \\
\hline 4 & \multirow[t]{2}{*}{ Soybean } & Yavatmal & Gunj, Pusad & RBs4 \\
\hline 5 & & Akola & $\begin{array}{l}\text { Pulses Research Unit Dr. } \\
\text { P.D.K.V., Akola }\end{array}$ & RBs5 \\
\hline 6 & \multirow[t]{2}{*}{ Pigeonpea } & Yavatmal & Takli & RBs6 \\
\hline 7 & & Nagpur & Katol & RBs7 \\
\hline 8 & \multirow[t]{2}{*}{ Sorghum } & Amravati & Tivasa & RBs8 \\
\hline 9 & & Nagpur & Umred & RBs9 \\
\hline 10 & \multirow[t]{2}{*}{ Chickpea } & Buldhana & Sangrampur & RBs10 \\
\hline 11 & & Akola & Umra & RBs11 \\
\hline 12 & \multirow[t]{2}{*}{ Green gram } & Amravati & Pipalkhutta & RBs12 \\
\hline 13 & & Buldhana & Jalgaon jamod & RBs13 \\
\hline 14 & \multirow[t]{2}{*}{ Paddy } & Chandrapur & Sindewahi & RBs14 \\
\hline 15 & & Gadchiroli & Allapalli & RBs15 \\
\hline
\end{tabular}


Table.4 Details of diseased sample used for isolation of fungal pathogens

\begin{tabular}{|c|c|c|l|}
\hline Sr.No. & Crops & Diseased samples used for isolation & \multicolumn{1}{|c|}{ Fungal pathogens Isolated } \\
\hline $\mathbf{1}$ & Cotton & Leaves & Myrothecium roridum \\
\hline $\mathbf{2}$ & Cotton & Leaves & Alternaria alternate \\
\hline $\mathbf{3}$ & Pigeonpea & Roots & Fusarium udum \\
\hline $\mathbf{4}$ & Soybean & Leaves & Alternaria alternate \\
\hline $\mathbf{5}$ & Soybean & Leaves & Colletotrichum truncatum \\
\hline $\mathbf{6}$ & Soybean & Roots & Rhizoctonia bataticola \\
\hline $\mathbf{7}$ & Sorghum & Seed & Curvularia lunata \\
\hline $\mathbf{8}$ & Sorghum & Seed & Fusarium moniliformae \\
\hline $\mathbf{9}$ & Chickpea & Roots & Fusarium oxysporum f.sp. ciceri \\
\hline $\mathbf{1 0}$ & Chickpea & Roots & Sclerotium rolfsii \\
\hline $\mathbf{1 1}$ & Green gram & Leaves & Phoma medicaginis \\
\hline $\mathbf{1 2}$ & Paddy & Stem & Rhizoctonia solani \\
\hline $\mathbf{1 3}$ & Bean & Leaves & Colletotrichum dematium \\
\hline
\end{tabular}

Table.5 Efficacy of phylloplane Bacillus subtilis isolates against foliar fungal pathogens by dual culture technique

\begin{tabular}{|c|c|c|c|c|c|c|c|c|c|c|c|c|c|c|c|}
\hline \multirow{2}{*}{\multicolumn{2}{|c|}{ B.subtilis host and isolates }} & \multicolumn{14}{|c|}{ Foliar fungal pathogens and host } \\
\hline & & \multicolumn{2}{|c|}{ M. roridum (Cotton) } & \multicolumn{2}{|c|}{ C. dematium (Bean) } & \multicolumn{2}{|c|}{ A. alternata (Soybean) } & \multicolumn{2}{|c|}{ C. truncatum (Soybean) } & \multicolumn{2}{|c|}{ C. lunata (Sorghum) } & \multicolumn{2}{|c|}{$\begin{array}{l}\text { P. medicaginis } \\
\text { (Green gram) }\end{array}$} & \multicolumn{2}{|c|}{ A.alternata (Cotton) } \\
\hline Host & Isolates & $\begin{array}{c}\text { Av.radial } \\
\text { mycelial } \\
\text { growth } \\
(\mathbf{m m})\end{array}$ & $\begin{array}{c}\text { Per cent } \\
\text { growth } \\
\text { inhibition }\end{array}$ & $\begin{array}{c}\text { Av.radial } \\
\text { mycelial } \\
\text { growth } \\
(\mathbf{m m})\end{array}$ & $\begin{array}{c}\text { Per cent } \\
\text { growth } \\
\text { inhibition }\end{array}$ & $\begin{array}{c}\text { Av.radial } \\
\text { mycelial } \\
\text { growth } \\
(\mathbf{m m})\end{array}$ & $\begin{array}{c}\text { Per cent } \\
\text { growth } \\
\text { inhibition }\end{array}$ & $\begin{array}{c}\text { Av.radial } \\
\text { mycelial } \\
\text { growth } \\
(\mathbf{m m})\end{array}$ & $\begin{array}{l}\text { Per cent } \\
\text { growth } \\
\text { inhibition }\end{array}$ & $\begin{array}{c}\text { Av.radial } \\
\text { mycelial } \\
\text { growth } \\
(\mathbf{m m})\end{array}$ & $\begin{array}{l}\text { Per cent } \\
\text { growth } \\
\text { inhibition }\end{array}$ & $\begin{array}{c}\text { Av.radial } \\
\text { mycelial } \\
\text { growth } \\
(\mathbf{m m})\end{array}$ & $\begin{array}{l}\text { Per cent } \\
\text { growth } \\
\text { inhibition }\end{array}$ & $\begin{array}{l}\text { Av.radial } \\
\text { mycelial } \\
\text { growth } \\
(\mathrm{mm})\end{array}$ & $\begin{array}{l}\text { Per cent } \\
\text { growth } \\
\text { inhibition }\end{array}$ \\
\hline \multirow[t]{2}{*}{ Cotton } & PBs1 & 16.00 & 80.40 & 11.83 & 79.18 & 30.17 & 47.68 & 22.00 & 61.85 & 31.50 & 62.19 & 28.00 & 57.36 & 32.83 & 62.12 \\
\hline & PBs2 & 15.00 & 81.63 & 10.67 & 81.22 & 29.17 & 49.41 & 20.83 & 63.88 & 28.83 & 65.40 & 28.67 & 56.34 & 22.67 & 73.84 \\
\hline \multirow[t]{3}{*}{ Soybean } & PBs3 & 10.37 & 87.30 & 10.17 & 82.10 & 21.50 & 62.71 & 19.17 & 66.75 & 32.33 & 61.20 & 20.83 & 68.28 & 22.33 & 74.23 \\
\hline & PBs4 & 15.33 & 81.22 & 11.83 & 79.18 & 25.50 & 55.78 & 21.17 & 63.29 & 33.17 & 60.19 & 21.17 & 67.76 & 27.33 & 68.46 \\
\hline & PBs5 & 16.17 & 80.20 & 12.33 & 78.30 & 24.33 & 57.81 & 20.33 & 64.74 & 31.00 & 62.79 & 26.00 & 60.40 & 28.00 & 67.69 \\
\hline \multirow{2}{*}{$\begin{array}{l}\text { Pigeon- } \\
\text { pea }\end{array}$} & PBs6 & 15.67 & 80.81 & 12.17 & 78.58 & 30.50 & 47.11 & 22.17 & 61.55 & 31.33 & 62.40 & 28.50 & 56.60 & 23.20 & 73.23 \\
\hline & PBs7 & 15.33 & 81.22 & 13.50 & 76.24 & 32.17 & 44.21 & 19.33 & 66.48 & 32.67 & 60.79 & 21.17 & 67.76 & 30.50 & 64.80 \\
\hline \multirow[t]{2}{*}{ Sorghum } & PBs8 & 15.50 & 81.02 & 13.17 & 76.82 & 34.17 & 40.74 & 21.17 & 63.29 & 25.00 & 69.99 & 28.17 & 57.10 & 29.50 & 65.96 \\
\hline & PBs9 & 14.50 & 82.24 & 12.33 & 78.30 & 32.17 & 44.21 & 22.50 & 60.98 & 26.67 & 67.99 & 29.17 & 55.58 & 29.50 & 65.96 \\
\hline \multirow{2}{*}{$\begin{array}{l}\text { Green } \\
\text { gram }\end{array}$} & PBs10 & 13.33 & 83.67 & 12.50 & 78.00 & 32.17 & 44.21 & 24.50 & 57.51 & 31.33 & 62.40 & 27.00 & 58.88 & 30.67 & 64.61 \\
\hline & PBs11 & 14.00 & 82.85 & 12.00 & 78.88 & 29.50 & 48.84 & 21.00 & 63.58 & 33.50 & 59.79 & 27.17 & 58.62 & 31.50 & 63.65 \\
\hline \multirow[t]{2}{*}{ Paddy } & PBs12 & 15.00 & 81.63 & 11.50 & 79.76 & 26.33 & 54.34 & 18.33 & 68.21 & 33.50 & 59.79 & 31.00 & 52.79 & 30.33 & 65.00 \\
\hline & PBs13 & 12.00 & 85.30 & 10.33 & 81.82 & 10.33 & 82.08 & 12.50 & 78.32 & 12.00 & 85.59 & 15.67 & 76.13 & 18.67 & 78.45 \\
\hline \multirow[t]{6}{*}{ Chickpea } & PBs14 & 17.00 & 79.18 & 11.83 & 79.18 & 29.17 & 49.41 & 21.50 & 62.71 & 26.83 & 67.80 & 22.50 & 65.73 & 25.33 & 70.77 \\
\hline & PBs15 & 17.50 & 78.57 & 33.17 & 41.63 & 31.83 & 44.80 & 20.17 & 65.02 & 26.33 & 68.40 & 29.33 & 55.33 & 30.50 & 64.80 \\
\hline & Control & 81.67 & 00.00 & 56.83 & 00.00 & 57.67 & 00.00 & 57.67 & 00.00 & 83.33 & 00.00 & 65.67 & 00.00 & 86.67 & 00.00 \\
\hline & F Test & Sig. & - & Sig. & - & Sig. & - & Sig. & - & Sig. & - & Sig. & - & Sig. & - \\
\hline & $\operatorname{SE}(m) \pm$ & 0.58 & - & 0.59 & - & 0.89 & - & 1.00 & - & 0.73 & - & 0.48 & - & 0.52 & - \\
\hline & $\mathrm{CD}(\mathrm{P}=0.01)$ & 2.26 & - & 2.34 & - & 3.34 & - & 3.89 & - & 2.81 & - & 1.87 & - & 1.92 & - \\
\hline
\end{tabular}


Table.6 Efficacy of phylloplane Bacillus subtilis isolates against foliar fungal pathogens by dual culture technique (mean of mycelial growth and inhibition)

\begin{tabular}{|c|c|c|c|c|}
\hline B. subtilis Isolates & Locations & Host of B.subtilis isolates & $\begin{array}{l}\text { Mean of mycelial growth of } \\
\text { fungi tested }(\mathrm{mm})\end{array}$ & $\begin{array}{l}\text { Mean of mycelial growth } \\
\text { inhibition of fungi tested }(\%)\end{array}$ \\
\hline PBs1 & Hinganghat & Cotton & 24.61 & 64.39 \\
\hline PBs2 & Murali & Cotton & 22.26 & 67.38 \\
\hline PBs3 & Amkheda & Soybean & 19.52 & 71.79 \\
\hline PBs4 & Brahamanwada & Soybean & 22.21 & 67.98 \\
\hline PBs5 & Yavatmal & Soybean & 22.59 & 67.41 \\
\hline PBs6 & Ganeshpur & PigeonPea & 23.36 & 65.75 \\
\hline PBs7 & Doangaon & PigeonPea & 23.52 & 65.92 \\
\hline PBs8 & Akola sorghum & Sorghum & 23.81 & 64.98 \\
\hline PBs9 & RRC, Amravati & Sorghum & 23.83 & 65.03 \\
\hline PBs 10 & Patur & Green gram & 24.50 & 64.18 \\
\hline PBs11 & Varud & Green gram & 24.09 & 65.17 \\
\hline PBs12 & Sakoli & Paddy & 23.71 & 65.93 \\
\hline PBs13 & Amgaon & Paddy & 13.07 & 81.09 \\
\hline PBs14 & Akola & Chickpea & 22.02 & 67.82 \\
\hline PBs 15 & Dhanora & Chickpea & 26.97 & 59.79 \\
\hline Control & - & - & 69.93 & 00.00 \\
\hline
\end{tabular}


Table.7 Efficacy of rhizospheric Bacillus subtilis isolates against soil borne fungal pathogens by dual culture technique

\begin{tabular}{|c|c|c|c|c|c|c|c|c|c|c|c|c|c|}
\hline \multirow{2}{*}{\multicolumn{2}{|c|}{$\begin{array}{c}\text { B.subtilis host and } \\
\text { isolates }\end{array}$}} & \multicolumn{12}{|c|}{ Soil borne fungal pathogens and host } \\
\hline & & \multicolumn{2}{|c|}{$\begin{array}{c}\text { F. udum } \\
\text { (Pigeonpea) }\end{array}$} & \multicolumn{2}{|c|}{$\begin{array}{l}\text { F. moniliformae } \\
\text { (Sorghum) }\end{array}$} & \multicolumn{2}{|c|}{$\begin{array}{c}\text { F. oxysporum f.sp. } \\
\text { ciceri (Chickpea) }\end{array}$} & \multicolumn{2}{|c|}{$\begin{array}{c}\text { S. rolfsii } \\
\text { (Chickpea) }\end{array}$} & \multicolumn{2}{|c|}{$\begin{array}{l}\text { R. bataticola } \\
\text { (Soybean) }\end{array}$} & \multicolumn{2}{|c|}{$\begin{array}{l}\text { R. solani } \\
\text { (Rice) }\end{array}$} \\
\hline Host & Isolates & $\begin{array}{c}\text { Av.radial } \\
\text { mycelial } \\
\text { growth } \\
(\mathbf{m m})\end{array}$ & $\begin{array}{l}\text { Per cent } \\
\text { growth } \\
\text { inhibition }\end{array}$ & $\begin{array}{c}\text { Av.radial } \\
\text { mycelial } \\
\text { growth } \\
(\mathbf{m m})\end{array}$ & $\begin{array}{l}\text { Per cent } \\
\text { growth } \\
\text { inhibition }\end{array}$ & $\begin{array}{c}\text { Av.radial } \\
\text { mycelial } \\
\text { growth } \\
(\mathbf{m m})\end{array}$ & $\begin{array}{l}\text { Per cent } \\
\text { growth } \\
\text { inhibition }\end{array}$ & $\begin{array}{c}\text { Av.radial } \\
\text { mycelial } \\
\text { growth } \\
(\mathbf{m m})\end{array}$ & $\begin{array}{l}\text { Per cent } \\
\text { growth } \\
\text { inhibition }\end{array}$ & $\begin{array}{c}\text { Av.radial } \\
\text { mycelial } \\
\text { growth } \\
(\mathbf{m m})\end{array}$ & $\begin{array}{l}\text { Per cent } \\
\text { growth } \\
\text { inhibition }\end{array}$ & $\begin{array}{c}\text { Av.radial } \\
\text { mycelial } \\
\text { growth } \\
(\mathbf{m m})\end{array}$ & $\begin{array}{l}\text { Per cent } \\
\text { growth } \\
\text { inhibition }\end{array}$ \\
\hline \multirow[t]{3}{*}{ Cotton } & RBs1 & 33.50 & 52.48 & 31.50 & 56.55 & 31.83 & 56.39 & 36.33 & 59.63 & 40.50 & 55.00 & 33.50 & 62.77 \\
\hline & RBs2 & 30.17 & 57.20 & 21.20 & 70.76 & 26.33 & 63.93 & 25.33 & 71.85 & 33.67 & 62.56 & 25.83 & 71.30 \\
\hline & RBs3 & 34.63 & 50.87 & 28.50 & 60.68 & 29.17 & 60.04 & 28.00 & 68.88 & 41.50 & 53.88 & 35.33 & 60.74 \\
\hline \multirow[t]{2}{*}{ Soybean } & RBs4 & 35.33 & 49.88 & 31.50 & 56.55 & 30.33 & 58.45 & 34.67 & 61.47 & 44.67 & 50.36 & 40.67 & 54.81 \\
\hline & RBs5 & 36.33 & 48.46 & 29.83 & 58.85 & 32.83 & 55.02 & 34.17 & 62.03 & 46.50 & 48.33 & 40.50 & 55.00 \\
\hline \multirow{2}{*}{$\begin{array}{l}\text { Pigeon- } \\
\text { pea }\end{array}$} & RBs6 & 34.83 & 50.59 & 30.33 & 58.16 & 36.00 & 50.68 & 35.17 & 60.92 & 42.83 & 52.41 & 41.17 & 54.25 \\
\hline & RBs7 & 36.00 & 48.93 & 30.00 & 58.62 & 31.33 & 57.08 & 37.00 & 58.88 & 43.17 & 52.03 & 40.50 & 55.00 \\
\hline \multirow[t]{2}{*}{ Sorghum } & RBs8 & 36.83 & 47.75 & 32.33 & 55.40 & 36.83 & 49.54 & 38.33 & 57.41 & 43.67 & 51.47 & 41.50 & 53.88 \\
\hline & RBs9 & 37.00 & 47.51 & 32.17 & 55.62 & 32.83 & 55.02 & 37.50 & 58.33 & 45.17 & 49.81 & 41.17 & 54.25 \\
\hline \multirow[t]{2}{*}{ Chickpea } & RBs10 & 34.60 & 50.92 & 33.00 & 54.48 & 36.17 & 50.45 & 32.67 & 63.70 & 43.00 & 52.22 & 42.67 & 52.58 \\
\hline & RBs11 & 37.17 & 47.27 & 33.83 & 53.33 & 32.17 & 55.93 & 35.20 & 60.88 & 35.50 & 60.55 & 42.17 & 53.14 \\
\hline \multirow{2}{*}{$\begin{array}{l}\text { Green } \\
\text { gram }\end{array}$} & RBs12 & 31.50 & 55.31 & 23.50 & 67.59 & 29.33 & 59.82 & 26.07 & 71.03 & 37.67 & 58.11 & 32.67 & 63.70 \\
\hline & RBs13 & 32.17 & 54.36 & 22.50 & 68.96 & 29.50 & 59.58 & 26.83 & 70.18 & 34.83 & 61.30 & 31.50 & 65.00 \\
\hline \multirow[t]{6}{*}{ Paddy } & RBs14 & 33.00 & 53.19 & 23.00 & 68.27 & 30.50 & 58.21 & 27.83 & 69.07 & 34.67 & 61.47 & 31.67 & 64.81 \\
\hline & RBs15 & 36.17 & 48.69 & 32.33 & 55.40 & 31.83 & 56.39 & 36.50 & 59.44 & 42.33 & 52.96 & 39.33 & 56.30 \\
\hline & Control & 70.50 & 00.00 & 72.50 & 00.00 & 73.00 & 00.00 & 90.00 & 00.00 & 90.00 & 00.00 & 90.00 & 00.00 \\
\hline & F Test & Sig. & - & Sig. & - & Sig. & - & Sig. & - & Sig. & - & Sig. & - \\
\hline & $\mathrm{SE}(\mathrm{m}) \pm$ & 0.04 & - & 0.07 & - & 0.06 & - & 0.12 & - & 0.09 & - & 0.05 & - \\
\hline & $\mathrm{CD}(\mathrm{P}=0.01)$ & 0.015 & - & 0.27 & - & 0.26 & - & 0.34 & - & 0.35 & - & 0.21 & - \\
\hline
\end{tabular}


Table.8 Efficacy of rhizospheric Bacillus subtilis isolates against soil borne fungal pathogens by dual culture technique (mean of mycelial growth and inhibition)

\begin{tabular}{|c|c|c|c|c|}
\hline $\begin{array}{l}\text { B.subtilis } \\
\text { Isolates }\end{array}$ & Location & $\begin{array}{l}\text { Host of B. subtilis } \\
\text { isolates }\end{array}$ & $\begin{array}{l}\text { Mean of mycelial growth } \\
\text { of fungi tested (mm) }\end{array}$ & $\begin{array}{c}\text { Mean of mycelial growth } \\
\text { inhibition of fungi tested }(\%)\end{array}$ \\
\hline RBs1 & Paratwada & Cotton & 34.52 & 57.13 \\
\hline RBs2 & Bhadgaon & Cotton & 28.13 & 65.00 \\
\hline RBs3 & COA, Nagpur & Cotton & 32.85 & 59.18 \\
\hline RBs4 & Gunj, Pusad & Soybean & 36.19 & 55.25 \\
\hline RBs5 & Akola & Soybean & 36.69 & 54.61 \\
\hline RBs6 & Takli & Pigeonpea & 36.72 & 54.50 \\
\hline RBs7 & Katol & Pigeonpea & 36.33 & 55.09 \\
\hline RBs8 & Tivasa & Sorghum & 38.16 & 52.57 \\
\hline RBs9 & Umred & Sorghum & 37.64 & 53.42 \\
\hline RBs10 & Sangrampur & Chickpea & 37.01 & 54.05 \\
\hline RBs11 & Akot & Chickpea & 36.00 & 55.18 \\
\hline $\mathrm{RBs} 12$ & Pipalkhutta & Green gram & 29.06 & 63.87 \\
\hline RBs13 & Jalgaon jamod & Green gram & 29.55 & 63.23 \\
\hline RBs14 & Sindewahi & Rice & 30.11 & 62.50 \\
\hline RBs15 & Allapalli & Rice & 36.41 & 54.86 \\
\hline Control & - & - & 81.00 & 00.00 \\
\hline
\end{tabular}


Similar findings in respect of mycelial growth inhibitions of Curvularia geniculata with antifungal compound produced by $B$. subtilis was reported by Dass and Teyegaga (1996). The strong antifungal effect in vitro against Phoma spp. with B. subtilis, isolated from wheat phylloplane and suppression of Myrothecium spp. in watermelon with $B$. pumilus were recorded by Perello et al.(2001) and Lokesh et al., (2007) respectively. The findings reported by different workers regarding antifungal activity of $B$. subtilis against tested fungi confirmed the results of present investigations.

\section{Efficacy of rhizospheric Bacillus subtilis isolates against soil borne fungal pathogens by dual culture technique}

Data presented in the Table 7 and 8 indicated that all the isolates suppress the growth of all tested soil borne fungal pathogens. Mycelial growth inhibition ranges between 57.16 $71.89 \%$ in RBs2 isolates. Minimum mycelial growth $(28.13 \mathrm{~mm})$ with maximum mycelial growth inhibition (65.00\%) of Fusarium udum, Fusarium moniliformae, Fusarium oxysporum f.sp. ciceri, Sclerotium rolfsii, Rhizoctonia bataticola and Rhizoctonia solani was recorded in $B$. subtilis RBs2 followed by RBs12 (63.60\%), RBs13 (63.35\%) and RBs14 (63.08\%) isolates. The other isolates of B. subtilis i.e. RBs8, RBs9, RBs10 and RBs6 were found least effective against all the tested soil borne fungal pathogens.

The results of present investigation are corroborates the findings of Wijesundera and Herath (1994) who isolated Bacillus subtilis from rice phylloplane, which was inhibitory to $R$. solani causal agent of sheath blight of rice. Karimi et al., (2012) recorded antagonistic effects of six rhizosphere isolates of Pseudomonas and Bacillus spp. obtained from chickpea against Fusarium oxysporum f. sp. ciceris as potential biocontrol agents in vitro. Jadhav et al., (2014) evaluated five isolates of Bacillus subtilis from rhizospheric soil of wilt infected pigeonpea plants, viz. $\mathrm{BS} 1, \mathrm{BS} 2, \mathrm{BS} 3, \mathrm{BS} 4, \mathrm{BS} 5$. Isolate BS5 was found most effective against Fusarium udum which recorded $48.12 \%$ mycelial growth inhibition in dual culture technique. AbouAly HE et al., (2015) revealed that among 116 bacterial isolates, nineteen bacterial isolates showed the highest inhibition against Fusarium oxysporum, Rhizoctonia solani and Sclerotium rolfsii. Isolates B103 and B38 showed considerable inhibition against pathogenic fungi. The most potent isolates for bioagent production were chosen and were identified as Pseudomonas fluorescens (B103) and Bacillus subtilis (B38).

Rajkumar et al., (2018) screened thirty $B$. subtilis isolates in vitro against $S$. rolfsii, isolate BS16 inhibited maximum mycelial growth $(64.04 \%)$ followed by BS 30 (11.98 $\%)$. Shifa et al., (2015) recorded Bacillus subtilis strain G-1 was the most effective in inhibiting the mycelial growth of $S$. rolfsii. In vitro mycelial suppression of $R$. bataticola to the extent of $52.22 \%$ with B.subtilis was also reported by Veenashri Jainapur et al., (2019). Thus present studies confirm the efficiency of the isolates under investigations.

\section{References}

Abdallah A.B.R., H. J. Khiareddine, S. M. Tlili, A. Nefzi and S. M. Saidana, 2015. Endophytic Bacillus spp. from wild solanaceae and their antifungal potential against Fusarium oxysporum f. sp. lycopersici elucidated using whole cells, filtrate cultures and organic extracts. J. Plant Pathol. Microbiol. 6: 324.

Abou-Aly, H. E., N. A. Neweigy, R. A. Zaghloul, S. A. El-Sayed and A. M. Bahloul, 2015. Evaluation of some biocontrol agents against soil pathogenic fungi. Research Journal of 
Pharmaceutical. Biological and Chemical Sciences 6(1):639-647.

Adebanjo, A. and S. A. Bankole, 2004. Evaluation of some fungi and bacteria for biocontrol of anthracnose disease of cowpea. Journal of Basic Microbiology, 44(1):3-9.

Amaresan, N., V. Jayakumar, K. Kumar and N. Thajuddin, 2012. Isolation and characterization of plant growth promoting endophytic bacteria and their effect on tomato (Lycopersicon esculentum) and chilli (Capsicum annuum) seedling growth. Annals of Microbiology, 62(2):805-810.

Amin M., Z. Rakhisi and A. Z. Ahmady, 2015. Isolation and identification of Bacillus species from soil and evaluation of their antibacterial properties. Avicenna. J. Clin. Microb. Infec., 2(1): 23-33.

Ashwini Narasimhan and Srividya Shivakumar, 2016. Biocontrol of Rhizoctonia solani root rot of chilli by Bacillus subtilis formulations under pot conditions. Journal of Biological Control, 30(2): 109-118.

Berg, G., Fritze, A., Roskot, N. and Smalla, K. (2001) Evaluation of Potential Biocontrol Rhizobacteria from Different Host Plants of Verticillium dahliae Kleb. Journal of Applied Microbiology, 91, 963-971. https

Brian, B. McSpadden Gardener, 2004. Ecology of Bacillus and PaeniBacillus spp. in Agricultural System. The American Phytopathological Society, Vol.94 (9):1252-1258.

Cazorla, F.M., Romero, D., Perez-Garcia, A., Lugtenberg, B.J., Vicente, A.D. and Bloemberg, G. (2007) Isolation and Characterization Of antagonistic Bacillus subtilis Strains from the Avocado Rhizoplane Displaying Biocontrol Activity. Journal of Applied Microbiology, 103, 1950-1959
Dass, C. and A. Teyegaga, 1996. Growth suppression of some wood decay and other fungi by Bacillus subtilis. Australian J. Botany, 44(6): 705-712.

Jadhav, R. R., G. K. Giri and Y. K. Belkar, 2014. Characterization of Bacillus subtilis and management of wilt of pigeonpea caused by Fusarium udum. J. Pl. Dis. Sci. Vol. 9(2):180-184.

Karimi, K., J. Amini, B. Harighi and B. Bahramnejad, 2012. Evaluation of biocontol potential of Pseudomonas and Bacillus spp. against Fusarium wilt of chickpea. AJCS, 6(4): 695-703.

Karthick, M., C. Gopalakrishnan, E. Rajeswari and V. Karthik Pandi, 2017.In vitro efficacy of Bacillus spp. against Fusarium oxysporum f. sp. ciceri, the causal agent of Fusarium wilt of chickpea. Int. J. Curr. Microbiol. App. Sci. Vol. 6 (11):2751-2756.

Killani, A. S., R. C. Abaidoo, A. K. Akintokun, and M. A. Abiala, 2011. Antagonistic effect of indigenous Bacillus subtilis on root-/soil-borne fungal pathogens of cowpea. Researcher, 3(3).

Laha, G. S., S. Venkataraman, 2001. Sheath blight management in rice with biocontrol agents. Indian Phytopath., 54(4): 461-464..

Lokesh, S., B. G. Bharath, V. B. Raghavendra and M. Govindappa, 2007. Importance of plant growth-promoting rhizobacteria in enhancing the seed germination and growth of watermelon attacked by fungal pathogens. Acta Agronomica Hungarica, 55(2):243-249.

Mardanova, A. M., G. F. Hadieva, M. T. Lutfullin, I. V. Khilyas, L. F. Minnullina and A. G. Gilyazeva, L. M. Bogomolnaya and M. R. Sharipova, 2017. Bacillus subtilis strains with antifungal activity against the phytopathogenic fungi. Agricultural Sciences, 8:1-20. 
Patro, T. S. S. K., P. Bahadur and J. K. Jindal, 2002. Exploitation of antagonistic phylloplane bacteria for management of bacterial leaf spot of mungbean. Tropical Agricultural Research Vol. 14: 174-185.

Perello, A. M., R. Simon, M. Sisterna, C. Cordo and A. Arambarri, 2001. Microflora of wheat (Triticum aestivum L.) in buenos aires province (Argentina) and its possible significance in biological control of foliar pathogens. Zeitschrift fur Pflanzenkrankheiten und Pflanzenschutz, 108(5):459-471.

Prapagdee, B., L. Tharasaithong, R. Nanthaphot and C. Paisitwiroj, 2012. Efficacy of crude extract of antifungal compounds produced from Bacillus subtilis on prevention of anthracnose disease in Dendrobium orchid. Environment Asia, 5(1):32-38.

Rajkumar K., M. K. Naik, Y. S. Amaresh and G. Chennappa, 2018. In vitro screening of Bacillus subtilis isolates against Sclerotium rolfsii cause for collar rot of chilli, Int. J. Curr. Microbiol. App. Sci. Vol. 7(07):2687-2692.

Saha, D., G. D. Purkayastha, A. Ghosh, M. Isha and A. Saha, 2015. Isolation and characterization of two new Bacillus subtilis strains from the rhizosphere of eggplant as potential biocontrol agents. Journal of plant pathology 94(1):109118.

Shifa, H., C. Gopalakrishnan and R. Velazhahan, 2015. Efficacy of Bacillus subtilis G-1 in suppression of stem rot caused by Sclerotium rolfsii and growth promotion of groundnut. International
Journal of Agriculture, Environment and Biotechnology 8(1):111-118.

Smitha, K. P., E. Rajeswari, C. D. Alice and T. Raguchander, 2017. Evaluation of Bacillus subtilis for the management of dry root rot and vascular wilt of chickpea. Journal of Pharmacognosy and Phytochemistry 6(6): 967-970.

Tan, Z., Lin, B. and Zhang, R. (2013) A Novel Antifungal Protein of Bacillus subtilis B25. SpringerPlus, 2, 543

Theoduloz, C., A. Vega, M. Salazar, E. Gonza lez and L. Meza-Basso, 2003. Expression of a Bacillus thuringiensis d-endotoxin cry1Ab gene in Bacillus subtilis and Bacillus licheniformis strains that naturally colonize the phylloplane of tomato plants (Lycopersicon esculentum). Journal of Applied Microbiology, 94:375-381.

Veenashri Jainapur, Shalini N. Huilgol, S. M. Vastrad and R. B. Jolli, 2019. Antagonistic activity of biocontrol agents against Rhizoctonia bataticola causing dry root rot (DRR) in chickpea. Int. J. Curr. Microbiol. App. Sci., 8(2):1591-1595.

Wijesundera, R.L.C. and W.H.W.W. Herath, 1994. An isolates of Bacillus subtilis from Srilanka inhibitory to $R$. solani. J. Nation Science Council of Srilanka, 22(1): 15-21.

Wu, L., Wu, H.J., Qiao, J., Gao, X. and Borriss, R. (2015) Novel Routes for Improving Biocontrol Activity of Bacillus Based Bioinoculants. Frontiers in Microbiology, 6, 1395

\section{How to cite this article:}

Kendre, V. P., G. K. Giri and Mane, S. S. 2020. Evaluation of Antagonistic Potential of Bacillus subtilis against Plant Pathogenic Fungi. Int.J.Curr.Microbiol.App.Sci. 9(10): 19571968. doi: https://doi.org/10.20546/ijcmas.2020.910.239 